\title{
Comportamiento productivo de cuyes (Cavia porcellus) de engorde suplementados con tres niveles de butirato de sodio en la dieta
}

\author{
Productive performance of guinea pigs (Cavia porcellus) supplemented with \\ three levels of sodium butyrate in the diet
}
Isabel Guzmán G. ${ }^{1}$, Fernando Carcelén C. ${ }^{1,4}$, Miguel Ara G. ${ }^{1}$, Ronald Jiménez A. ${ }^{2}$, Sandra Bezada Q. ${ }^{1}$, Jorge Guevara V. ${ }^{3}$, Ana Asencios M. ${ }^{1}$

\section{Resumen}

El objetivo del presente estudio fue evaluar el efecto de la suplementación de butirato de sodio (BS) en la dieta sobre el comportamiento productivo de cuyes de engorde. Cincuenta cuyes machos destetados, de 14 a 21 días de edad, fueron distribuidos al azar en cinco tratamientos $(0,100,200,300$ ppm de BS, 200 ppm de Zn-Bacitracina). Se determinó la ganancia de peso, el consumo de alimento y la conversión alimenticia a los 70 días de la suplementación. No se detectaron patrones de respuesta por efecto del BS ni de ZnBacitracina. Se concluye que, bajo las condiciones experimentales, la suplementación con $\mathrm{BS}$ no mejoró los parámetros productivos evaluados.

Palabras clave: cuy; comportamiento productivo; parámetros productivos; butirato de sodio

\section{Abstract}

The aim of this study was to evaluate the effect of dietary supplementation with sodium butyrate (BS) on productive performance in fattening guinea pigs. Fifty 14-21 days old weaned male guinea pigs were randomly distributed in five treatments $(0,100$,

\footnotetext{
${ }^{1}$ Laboratorio de Bioquímica, Nutrición y Alimentación Animal, Facultad de Medicina Veterinaria, Universidad Nacional Mayor de San Marcos, Lima, Perú

${ }^{2}$ Estación Experimental del Centro de Investigaciones IVITA - El Mantaro, Universidad Nacional Mayor de San Marcos, Junín, Perú

${ }^{3}$ Escuela Académico Profesional de Ingeniería Agroindustrial, Facultad de Química e Ingeniería Química, Universidad Nacional Mayor de San Marcos, Lima, Perú

${ }^{4}$ E-mail: nandodeme@gmail.com
}

Recibido: 31 de octubre de 2018

Aceptado para publicación: 10 de mayo de 2019 
200, 300 ppm of BS, 200 ppm of Zn-Bacitracin). Body weight gain, feed consumption and feed conversion were evaluated 70 days after supplementation. No response patterns were detected due to BS or Zn-Bacitracin treatments. It is concluded that, under the experimental conditions, supplementation with BS did not improve the productive parameters evaluated.

Key words: guinea pig; productive performance; productive parameters; sodium butyrate

\section{INTRODUCCIÓN}

La crianza de cuyes (Cavia porcellus) ha experimentado un crecimiento significativo durante los últimos años debido a su rusticidad, la calidad de su carne y la rentabilidad de la actividad. Sin embargo, los problemas sanitarios, particularmente las infecciones gastrointestinales como salmonelosis siguen causado problemas, reportándose prevalencias de hasta $76 \%$ en crianzas familiares (Aguilar et al., 2011) y de $47 \%$ en crianzas tecnificadas (Obregón et al., 2018).

Las granjas de cuyes, al igual que crianzas de otras especies, recurren al uso de antibióticos como promotores de crecimiento (APC) para contrarrestar los problemas de salud y mejorar el rendimiento productivo. Sin embargo, el continuo uso de los APC en la dieta de los animales puede traer como consecuencia la emergencia de microorganismos resistentes a medicamentos utilizados para el tratamiento y control de enfermedades en animales y humanos, así como el riesgo de residuos de los antimicrobianos en la carne (Cancho et al., 2000; Chesson, 2005; Wegener, 2005). Alternativas al uso de los APC son los ácidos orgánicos (Partanen y Mroz, 1999), los probióticos y prebióticos (Roberfroid, 1998), las enzimas (Bedford y Schulze, 1998) y los extractos de plantas (Kamel, 2001).

Los ácidos orgánicos (AO) tienen el potencial de mejorar el rendimiento productivo ejerciendo actividad antibacteriana al bajar el $\mathrm{pH}$ extracelular. Esto reduce la coloni- zación intestinal por E. coli, Salmonella spp y otros patógenos, los cuales en su mayoría prosperan en un $\mathrm{pH}$ neutro o ligeramente alcalino (Canibe et al., 2001; Franco et al., 2005). En su forma no disociada, los AO reducen el pH del citoplasma de la bacteria, obligándola a incrementar su gasto energético para mantener su equilibrio osmótico (Östling y Lindgren, 1993), ejerciendo una presión mecánica sobre la pared del microorganismo, determinando que eventualmente pueda estallar (Salmond et al., 1984). Los AO pueden considerarse sustancias seguras, ya que no abandonan el tracto digestivo y por ello no dejan residuos en los productos animales (Carro y Ranilla, 2002).

El ácido butírico ha sido usado en la nutrición parenteral pos-cirugía intestinal para estimular la recuperación del intestino en humanos (Tappenden et al., 2003). Este ácido, cuando está presente en el tracto intestinal, induce la producción de péptidos (Guilloteau et al., 2009), los cuales estimulan el desarrollo y la reparación del tracto intestinal a través de un aumento en la proliferación celular (Bartholome et al., 2004). Parece ser que en animales hay un modo similar de acción. $\mathrm{Hu}$ y Guo (2007) demostraron un aumento en el desarrollo de las vellosidades intestinales de pollos cuando el butirato de sodio se agregó en la dieta, y Vallejos et al. (2015) obtuvieron resultados positivos en el desarrollo intestinal de cuyes de engorde tras la suplementación con butirato de sodio. Es posible que el efecto del ácido butírico sobre el desarrollo intestinal se refleje en el desempeño productivo (Wang y Zhou, 2007; Panda et al., 2009; Herrera et al., 2011). 
El objetivo de este estudio fue evaluar el efecto de la suplementación con butirato de sodio en dietas de cuyes de engorde sobre el comportamiento productivo en términos de ganancia de peso, consumo de materia seca e índice de conversión alimenticia.

\section{Materiales y Métodos}

\section{Localización}

El estudio se llevó a cabo entre febrero y abril de 2013, en las instalaciones del Instituto Veterinario de Investigaciones Tropicales y de Altura (IVITA) de la Facultad de Medicina Veterinaria de la Universidad Nacional Mayor de San Marcos, ubicado en El Mantaro en Junín, Perú, a una altitud de 3320 msnm.

\section{Arreglo Experimental}

Cincuenta cuyes machos destetados, de 14 a 21 días de edad, provenientes del cruce de las líneas lecheros/prolíficas (LP) de la granja comercial del IVITA en Huancayo fueron ubicados en 50 pozas individuales de $0.7 \mathrm{x} 0.65 \mathrm{x} 0.5 \mathrm{~m}$, con piso de cemento y paredes de malla con bordes de madera. Las pozas fueron equipadas con dispensadoras fijas de malla y alambre galvanizado para el suministro de forraje y recipientes de arcilla de $0.5 \mathrm{~L}$ para el suministro de concentrado. Las pozas fueron previamente desinfectadas con amonio cuaternario y cal y se instaló una cama nueva de paja. La temperatura del galpón se mantuvo en $14{ }^{\circ} \mathrm{C}$ mediante el uso de cortinas.

Las pozas fueron distribuidas al azar en cinco tratamientos consistentes en la suplementación con 0, 100, 200 o 300 ppm de butirato de sodio (BS) o 200 ppm de ZincBacitracina a una dieta base. Esta estuvo compuesta de una mezcla forrajera de rye grass (Lolium multiflorum) cv. Tama y trébol rojo (Trifolium pratense) y afrechillo de trigo. La mezcla forrajera provino de un potrero con proporción de siembra rye grass/ trébol rojo de 3:1. El forraje fue ofrecido diariamente en un equivalente a $50 \%$ de peso vivo y contenía $20 \%$ de materia seca (MS), $18 \%$ de proteína cruda (PC) y 2.7 Mcal de energía digestible (ED) por kilogramo de MS. El afrecho de trigo contenía 90\% de MS, 16\% de PC y $2.65 \mathrm{Mcal}$ ED/kg MS (Vallejos et al., 2015). La proporción de forraje:afrechillo ofrecido fue de 80:20.

Se mezcló BS 30\% protegido (Gustor BP70, Norel Animal Nutrition, España) o Zinc-Bacitracina (Promozinb 10\%, Laboratorios CUSA, Perú) con el afrechillo de trigo usando una mezcladora de doble cinta con capacidad de $80 \mathrm{~kg}$. En primera instancia se hizo la mezcla con $5 \mathrm{~kg}$ de afrechillo y luego se agregó los $35 \mathrm{~kg}$ restantes (Behnke, 1992).

Los cuyes fueron criados durante 70 días. El manejo de los animales correspondió a los procedimientos acostumbrados para animales de engorde en la Granja de Cuyes del IVITA, a excepción de la ocupación individual de las jaulas, lo cual confirió completa independencia de alimentación y espacio vital.

\section{Mediciones}

Los animales fueron pesados semanalmente a las 09:00 antes de ofrecerles el alimento. La ganancia de peso (g) fue calculada como la diferencia entre el peso final y el peso inicial. Para la estimación del consumo de alimento, se pesó diariamente el forraje y el afrechillo de trigo ofrecidos y rechazados. La expresión en MS fue obtenida a través del secado en estufa por $48 \mathrm{~h}$ de muestras de $30 \mathrm{~g}$ de forraje y $15 \mathrm{~g}$ de afrechillo. El consumo de MS en $g$ fue estimado por las diferencias agregadas entre la MS de alimento ofrecida y la rechazada. El índice de conversión alimenticia (ICA) fue calculado como la relación entre el consumo total $(\mathrm{g})$ y la ganancia de peso $(\mathrm{g})$. Todas las pesadas se efectuaron usando una balanza electrónica con $0.5 \mathrm{~g}$ de sensibilidad. 
Cuadro 1. Valores promedio $(n=10)$ de ganancia de peso, consumo de alimento e índice de conversión alimenticia en cuyes destetados alimentados con dietas suplementadas con butirato de sodio y Zn-bacitracina durante 70 días

\begin{tabular}{lccccc}
\hline & Zn- & \multicolumn{4}{c}{ Butirato de sodio (ppm) } \\
\cline { 3 - 6 } & $\begin{array}{c}\text { Bacitracina } \\
(200 \mathrm{ppm})\end{array}$ & 0 & 100 & 200 & 300 \\
\hline Ganancia de peso $(\mathrm{g})$ & 674.8 & 657.4 & 699.8 & 707.6 & 715.6 \\
Consumo de alimento $(\mathrm{g})$ & 4638.5 & 4539.4 & 4818.8 & 4808.7 & 4822.6 \\
Conversión alimenticia & 6.9 & 6.9 & 7.0 & 6.9 & 6.8 \\
\hline
\end{tabular}

\section{Análisis Estadístico}

Las respuestas en ganancia de peso, consumo e ICA a los diferentes tratamientos fueron evaluadas por medio de un análisis de varianza (ANOVA) utilizando un diseño completamente al azar con cinco tratamientos y 10 repeticiones. El patrón lineal o cuadrático de respuesta al butirato de sodio y al $\mathrm{Zn}$ bacitracina fueron estimados mediante funciones lineales de las medias de tratamientos (contrastes). Las funciones aov y glht del programa R 3.4.3 (R Core Team, 2013) fueron usadas en el ANOVA y en la prueba de funciones lineales, respectivamente. Para todas las pruebas estadísticas se usó un nivel de significancia de 0.05 .

\section{Resultados y Discusión}

El Cuadro 1 muestra los valores promedio de ganancia de peso, consumo de alimento e índice de conversión alimenticia para los cinco tratamientos en estudio. No se evidenciaron patrones estadísticamente significativos de respuesta lineales ni cuadráticos a los niveles crecientes de BS ni al tratamiento con Zn-Bacitracina,

No se ha encontrado literatura publicada que evalúe el comportamiento productivo de cuyes suplementados con BS. No obs- tante, Carraro et al. (2005) evaluaron el consumo de alimento de conejos suplementados con 500, 1000 o 2000 ppm de BS por 70 días sin encontrar diferencias significativas con el grupo control. De manera similar, Scapinello et al. $(1999,2001)$ y Michelan et al. (2002) tampoco encontraron efectos significativos sobre la ganancia de peso, consumo de alimento y conversión alimenticia de conejos con dietas suplementadas con hasta $2 \%$ de ácido fumárico o $0.15 \%$ de una mezcla acidificante conteniendo ácido sórbico, fosfórico, láctico, fumárico y propiónico.

La adición de $\mathrm{BS}$ en dietas en aves de postura o en pollos de engorde mostró un aparente aumento en el consumo de alimento, pero sin efectos significativos en la respuesta productiva (Sánchez et al., 2011; Mansoub et al., 2011; Sayrafi et al., 2011). Flores y Ramos (2008), utilizaron dietas de pollos de engorde suplementadas con $0,1,2$ o $3 \mathrm{~kg}$ de ácido butírico (Citrozim-Na) por tonelada métrica de alimento durante seis semanas, obteniendo incrementos significativos en la ganancia de peso al inicio y fin del estudio, pero no en todo el ciclo de crecimiento ni en los demás componentes del comportamiento productivo. Por otro lado, Panda et al. (2009) suplementaron la dieta de pollos de engorde con ácido butírico demostrando mayores ganancias de peso al control y similares al APC a partir de $0.4 \%$ de ácido butírico. 
Weber y Kerr (2008) utilizaron BS a dosis de $0.05,0.1,0.2$ y $0.4 \%$ y tilosina como APC en la suplementación de lechones destetados, concluyendo que el BS no incrementa la ganancia de peso de los lechones, pero que puede regular las respuestas inflamatorias. Sin embargo, Piva et al. (2009) encontraron una mejora significativa en la ganancia de peso y el consumo total de alimento en lechones destetados durante las dos primeras semanas de la suplementación con 800 ppm BS.

La variabilidad del efecto de la adición de BS sobre el comportamiento productivo puede estar asociada a factores como el tipo de alimento, edad del animal y la dosis administrada. Esta última puede influenciar la capacidad acidificante del ácido orgánico de acuerdo a su constante de disociación y solubilidad (Papatsiros y Christodoulopoulos, 2011). Así mismo, el estado de maduración intestinal está asociado a la edad del animal, el cual cobra un papel importante en la asimilación de estos compuestos orgánicos (Partanen y Mroz, 1999).

Los efectos productivos de la inclusión del BS en la dieta de los cuyes en el presente estudio no han sido definitivos, pues la aparente mejora en el comportamiento productivo no fue estadísticamente significativa. Tres características de estos resultados llaman la atención: (i) la baja variabilidad (coeficientes de $10.0,6.8$ y $4.8 \%$ para ganancia de peso, consumo de alimento y conversión alimenticia, respectivamente), (ii) la relativa buena ganancia de peso del control (9.4 g/animal/día), y (iii) la ausencia de respuestas significativas a la suplementación con APC. Estas en conjunto sugieren que, bajo las condiciones experimentales del estudio, los animales no estuvieron en un estrés o desequilibrio microbiano intestinal por la ausencia de un «desafío de campo». Consecuentemente, no hubo oportunidad para que el BS exprese su efecto benéfico sobre el comportamiento productivo.
Literatura Citada

1. Aguilar G, Bustamante J, Bazán V, Falcón N. 2011. Diagnóstico situacional de la cría de cuyes en una zona de Cajamarca. Rev Inv Vet Perú 22: 9-14. doi: 10.15381/rivep.v22i1.113

2. Bartholome A, Albin D, Baker D, Holst J, Tappenden K. 2004. Supplementation of total parental nutrition with butyrate increases structural aspects of intestinal adaptation after an $80 \%$ jejunoileal resection in neonatal piglets. Jpen-Parenter Enter 28: 210-222. doi: 10.1177/0148607104028004210

3. Bedford M, Schulze H. 1998. Exogenous enzymes for pigs and poultry. Nutr Res Rev 11: 91-114. doi: 10.1079/ NRR19980007

4. Behnke DC. 1992. Como mezclar alimentos de calidad: perspectivas sobre uniformidad de mezclado. Soya Noticias 229: 6-12.

5. Cancho B, García M, Simal J. 2000. El uso de los antibióticos en la alimentación animal: perspectiva actual. Cienc Tecnol Aliment 3: 39-47. doi: 10.1080/ 11358120009487647

6. Canibe N, Steien SH, Overland M, Jensen BB. 2001. Effect of Kdiformate in starter diets on acidity, microbiota, and the amount of organic acids in the digestive tract of piglets, and on gastric alterations. J Anim Sci 79: 2123-2133. doi: 10.2527/2001.7982123x

7. Carraro L, Xiccato G, Trocino A, Radaelli G. 2010. Dietary supplementation of butyrate in growing rabbits. Ital J Anim Sci 4: 538-540.

8. Carro MD, Ranilla MJ. 2002. Los aditivos antibióticos promotores del crecimiento de animales: situación actual y posibles alternativas. Sitio Argentino de Produc-ción Animal. [Internet]. Disponible en: http://www.produc-cionanimal.com.ar/informacion_tecnica/invernada_promotor es_crecimiento/01aditivos_antibioticos_promotores.pdf 
9. Chesson A. 2005. Phasing out antibiotic additives in the EU: worldwide relevance for animal food production. In: The International Debate Conference for the Feed \& Food Chain - antimicrobial growth promoters: worldwide ban on the horizon? The Netherlands. [Internet]. Available in: http://www.bastiaansecommunication.com/rua2014/ boa_AGP.pdf

10. Flores J, Ramos W. 2008. Efecto de ácido butírico Citrozim-Na sobre los parámetros productivos en la producción de pollo de engorde. Tesis de Ingeniero Agrónomo. Honduras: Escuela Agrícola Panamericana Zamorano. $22 \mathrm{p}$.

11. Franco LD, Fondevila M, Lobera, M, Castrillo C. 2005. Effect of combinations of organic acids in weaned pig diets on microbial species of digestive tract contents and their response on digestibility. J Anim Physiol An N 89: 88-93. doi: 10.1111/j.1439-0396.2005.-00553.x

12. Guilloteau P, Zabielski R, David J, Blum J, Morisset J, Biernat M, Wolinski J, et al. 2009. Sodium-butyrate as a growth promoter in milk replacer formula for young calves. J Dairy Sci 92: 1038-1049. doi: 10.3168/jds.20081213

13. Herrera I, Posadas E, Sánchez E, Fuente B, Laparra J, González $E$. 2011. Efecto del butirato de sodio sobre algunos parámetros productivos de gallinas de postura en semilibertad. Vet México 42: 227-232.

14. Ни Z, Gио Y. 2007. Effects of dietary sodium butyrate supplementation on the intestinal morphological structure, absorptive function and gut flora in chickens. Anim Feed Sci Tech 132: 240249. doi: 10.1016/j.anifeedsci.-2006.03.017

15. Kamel C. 2001. Tracing modes of action and roles of plant extracts in nonrumiants. In: Recent advances in animal nutrition. UK: Nothingham Univ Press. p 151-165.
16. Mansoub N, Rahimpour K, Majedi L, Mohammad M, Zabihi M, Kalhori M. 2011. Effect of different level of butyric acid glycerides on performance and serum composition of broiler chickens. World J Zool 6: 179-182.

17. Michelan A, Scapinello C, Natali M, Furlan A, Sakaguti E, Faria $H$, Santolin M, et al. 2002. Utilização de probiotico, ácido orgânico e antibiótico em dietas para coelhos em crescimento: ensaio de digestibilidade, avaliação da morfometria intestinal e desempenho. Rev Bras Zootecn 31: 2227-2237. doi: 10.1590/S1516-35982002000900011

18. Obregón R, Serrano-Martínez E, Chauca L. 2018. Causas de mortalidad neonatal en cobayos (Cavia porcellus) durante la estación fría en el Instituto Nacional de Investigación Agraria, LimaPerú. Salud Tecnol Vet 2: 93-99. doi: 10.20453/stv.v6i2.3436

19. Ordoñez R. 1998. Efecto de dos niveles de proteína y fibra cruda en el alimento de cuyes (Cavia porcellus) en lactación y crecimiento. Tesis de Ingeniero Zootecnista. Lima: Univ. Nacional Agraria La Molina. 65 p.

20. Ostling CE, Lindgren SE. 1993. Inhibition of Enterobacteria and Lysteria growth by lactic, acetic and formic acids. J Appl Bacteriol 75: 18-24. doi: 10.1111/j.1365-2672.1993.tb03402.x

21. Panda A, Rao S, Raju M y Sunder G 2009. Effect of butyric acid on performance, gastrointestinal tract health and carcass characteristics in broiler chickens. Asian Austral J Anim 22: 10261031. doi: 10.5713/ajas.2009.80298

22. Papatsiros VG, Christodoulopoulos $\boldsymbol{G}$ 2011. The use of organic acids in rabbit farming. Online J Anim Feed Res 1: 434438.

23. Partanen KH, Mroz Z. 1999. Organic acids for performance enhancement in pig diets. Nutr Res Rev 12: 117-145. doi: 10.1079/095442299108728884 
24. Piva A, Morlacchini M, Casadei G, Gatta P, Biagi G, Prandini A. 2009. Sodium butyrate improves growth performance of weaned piglets during the first period after weaning. Ital J Anim Sci 1: 35-42. doi: 10.4081/ijas.2002.35

25. $R$ Core Team. 2013. R: a language and environment for statistical computing. $R$ Foundation for Statistical Computing. Vienna, Austria. URL http://www.Rproject.org/

26. Roberfroid M. 1998. Prebiotics and synbiotics: concepts and nutritional properties. Brit J Nutr 80: 197-202. doi: 10.1017/S0007114500006024

27. Salmond C, Kroll R, Booth I. 1984. The effect of food preservatives on $\mathrm{pH}$ homeostasis in Escherichia coli. J Gen Microbiol 130:2845-2850. doi: 10.1099/ 00221287-130-11-2845

28. Sánchez I, Posadas E, Sánchez E, Fuente B, Laparra JL, Avila E. 2011. Efecto de butirato de sodio sobre algunos parámetros productivos de gallinas de postura en semilibertad. Vet México 42:227-232.

29. Sayrafi, R., F. Soltanalinejad, $R$. Shahrooz, S. Rahimi, 2011. Comparative study of the effect of alternative and antibiotic feed additives on the performance and intestinal histomorphometrical parameters of broiler chickens. Afr J Agric Res 6: 27942799. doi:10.5897/AJAR10.1031

30. Scapinello C, Faria HG, Furlan AC. 1999. Influencia de diferentes níveis de ácido fumárico sobre o desempenho de coelhos em crescimento. Rev Bras Zootecn 28: 785-790. doi: 10.1590/ S1516-35981999000400019
31. Scapinello C, Faria HG, Furlan AC, Michelan AC. 2001. Efeito da utilização de oligossacarídeo manose e acidificantes sobre o desempenho de coelhos em crescimento. Rev Bras Zootecn 30: 1272-1277. doi: 10.1590/S151635982001000500021

32. Tappenden K, Albin D, Bartholome A, Mangian H. 2003. Glucagon-like peptide-2 and short-chain-fatty acids: a new twist to an old story. J Nutr 133: 3717-3720.

33. Vallejos D, Carcelén F, Jiménez, R, Perales R, Santillán G, Ara M, Quevedo $W$, et al. 2015. Efecto de la suplementación de butirato de sodio en la dieta de cuyes (Cavia porcellus) de engorde sobre el desarrollo de las vellosidades intestinales y criptas de lieberkühn. Rev Inv Vet Perú 26: 395-403. doi: 10.15381/ rivep.v26i3.11186

34. Wang J, Zhou H. 2007. Comparison of the effects of Chinese herbs, probiotics and prebiotics with those of antibiotics in diets on the performance of meat ducks. J Anim Feed Sci 16: 96103. doi: $10.22358 / \mathrm{jafs} / 66730 / 2007$

35. Weber TE, Kerr BJ. 2008. Effect of sodium butyrate on growth performance and response to lipopolysaccharide in weanling pigs. J Anim Sci 86: 442-450. doi: $10.2527 /$ jas.2007-0499

36. Wegener H. 2005. Use of antimicrobial growth promoters in food animals: the risks outweigh the benefits. In: Antimicrobial growth promoters: worldwide ban on the horizon? The Netherlands. [Internet]. Available in: http://www.bastiaanse-communication.com/rua2014/ boa_AGP.pdf 